\title{
A Vehicle Target Assignment Method
}

\author{
Yong Sun, Wenwei Li, Dan Huang and Yuan Feng \\ China Research and Development Academy of Machinery Equipment, Beijing, China
}

\begin{abstract}
Based on some definition of threat degree, shooting validity and gain, this paper propose a method of vehicle target assignment, the target can be assigned by rule or integer programming, this method is easy and practicality.
\end{abstract}

\section{Keywords- target assignment; vehicle; method}

\section{INTRODUCTION}

Before vehicle target assignment, multiple view of the observing and sighting instrument search, finding the target summary.

\section{The TARgET AsSIGNMENT DEFINITION (N TARGET, M FIRE UNIT)}

\section{A. The Threat Degree Aij of Target $i$ to Fire $j$}

$$
A_{i j}=\left\{\begin{array}{cc}
0 & \frac{R_{i}}{D_{i j}}<1 \\
\left(\frac{R_{i}}{D_{i j}}-1\right)^{2} \cdot \frac{1}{1+J_{i j}} \cdot L_{i} & \frac{R_{i}}{D_{i j}} \geq 1 \text { and enemy isn't guided weapon } \\
\frac{1}{1+J_{i j}} \cdot L_{i} & \frac{R_{i}}{D_{i j}} \geq 1 \text { and enemy is guided weapon }
\end{array}\right.
$$

$$
\mathrm{Ri} \text { — range of enemy fire } \mathrm{i}
$$

Dij__ i to j distance

$\mathrm{Jij}$ _ route shortcut of $\mathrm{i}$ to $\mathrm{j}$ (If the relative movement)

$\mathrm{Li}$ - the comprehensive value of out area of attack, the angle, the target weapon firing accuracy and the enemy projectile's damage ability to out fire. $0 \leq L_{i} \leq 1$

B. The Shooting Validity Bij of fire $j$ to Target $i$

$$
B_{i j}=\left\{\begin{array}{cc}
0 & \frac{r_{j}}{D_{i j}}<1 \\
I_{i j} \cdot\left(\frac{r_{j}}{D_{i j}}-1\right)^{2} \cdot \lambda^{k} \cdot L_{i}^{\prime} & \frac{r_{j}}{D_{i j}} \geq 1 \text { and we are not guided weapon } \\
I_{i j} \cdot \lambda^{k} \cdot L_{i}^{\prime} & \frac{r_{j}}{D_{i j}} \geq 1 \text { and we are guided weapon }
\end{array}\right.
$$

$$
I_{i j}=\left\{\begin{array}{c}
0 \\
\alpha \quad i \text { not in } j \text { defence area } \\
\alpha \quad i \text { in } j \text { defence area }
\end{array}\right.
$$

$$
0 \leqslant \alpha \leqslant 1
$$

$\mathrm{rj}$ _ the maximum range of fire $\mathrm{j}$

$\mathrm{k}$ - integer, when $\mathrm{j}$ has been assigned to $\mathrm{k}$ targets, the Bij value of the distribution is reduced by $\lambda^{k}, \lambda \in(0,1)$

$\mathrm{Li}$ - the comprehensive value of the target's distance coefficient, the target area of attack and the enemy's target anti damaging ability of different types (command car and common vehicle), $0 \leq L_{i}{ }^{\prime} \leq 1$

C. The Gain Cij of Fire unit j Shooting Target $i$

$$
C_{i j}=A_{i j} \cdot B_{i j}
$$

\section{ASSignMent By RULE}

\section{A. Assignment Criterion}

(1) When assignment start, target collection is E, fire unit collection is $\mathrm{F}$;

(2) Each fire unit $\mathrm{j}$ corresponding to a target set $\mathrm{Ej}$, initially empty set, the target in $\mathrm{Ej}$ is assigned to the fire unit;

(3) Each target i corresponding to a fire unit set Fi, that to be assigned, initially $\mathrm{F}$ is collection;

(4) Each target is first assigned to the largest gains fire unit;

(5) When the j-th fire unit is assigned to a target, the firing proceeds is adjusted $C_{i j}=\lambda C_{i j} \lambda \in(0,1)$, so as to avoid some of the units assigned to too many targets, while others are not assigned;

(6) If a target $\mathrm{i}, \max _{j}\left\{C_{i j}\right\}<\delta$, then the target temporarily not assigned (with the dynamic changes in the battlefield situation, the allocation will continue to be);

(7) When all objects are assigned one round, then do a second round of distribution, this time fire unit that has been 
assigned to the target $\mathrm{i}$ no longer participate in the distribution of i's (each target corresponds to a dynamic changes to be distributed fire unit set Fi);

(8) Stops assignment criterion: In addition to the target $\max _{j}\left\{C_{i j}\right\}<\delta$

, the rest of the target at least is assigned to a fire unit, in addition to the fire unit $\max _{i}\left\{C_{i j}\right\}<\delta$, the rest of the fire unit at least is assigned to a target, and the corresponding relationship number between the fire and the target is not less than $p$;

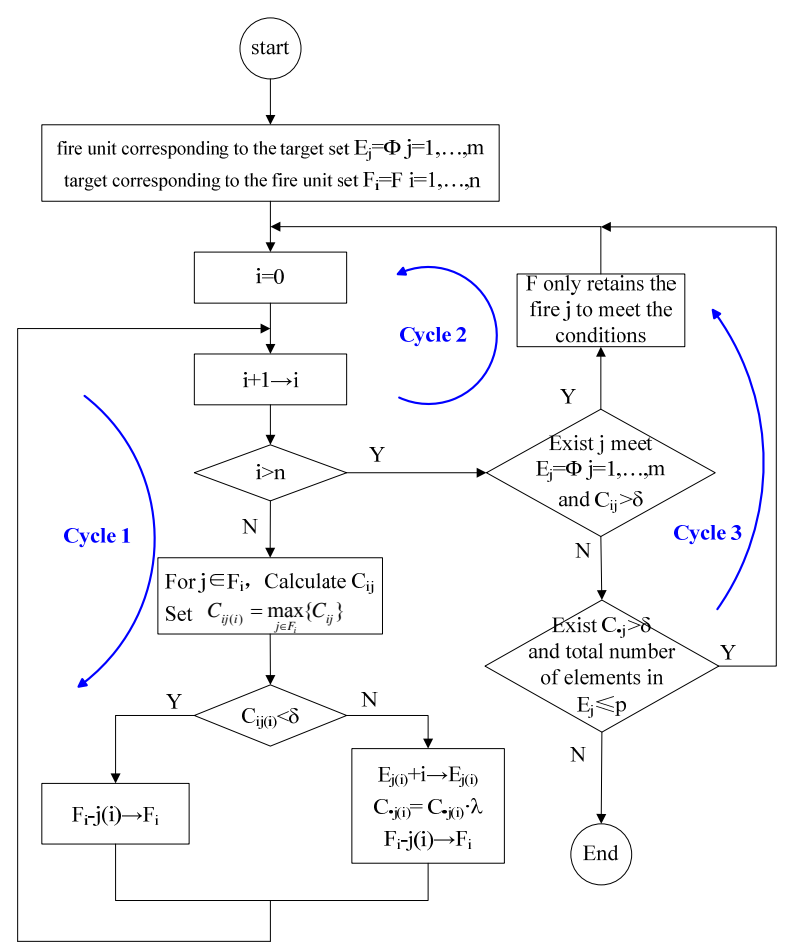

FIGURE I. DISTRIBUTION FLOWCHART.

Description: Wherein cycle 1 achieve each target assigned to at least one fire unit, cycle 2 achieve each fire unit assigned to at least one target, cycle 3 achieve multiple fire units attack a target and a fire unit to attack multiple targets, where $\mathrm{p}$ is $\max \{\mathrm{m}, \mathrm{n}\}+$ integer.

\section{TARgET AsSignMENT By INTEGER PROGRAMMING}

There are $n$ targets, $m$ fire units and $n \leq m$ (divided-period shooting different target, when the target distance, not assignment, to ensure $n \leq m$ )

Set

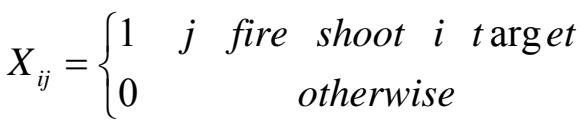

Then $\sum_{i=1}^{\mathrm{n}} X_{i j}=1 \_$a fire only attack one target

$\sum_{i=1}^{\mathrm{n}} \sum_{j=1}^{\mathrm{m}} X_{i j} \leq \mathrm{p}$ more than $\mathrm{p}$

Under the above two constraint conditions, find the maximum value of the following objective function $F$.

$$
F\left(X_{i j} \quad \begin{array}{l}
i=1 \cdots \mathrm{n} \\
j=1 \cdots \mathrm{m}
\end{array}\right)=\sum_{i=1}^{\mathrm{n}} \sum_{j=1}^{\mathrm{m}} X_{i j} C_{i j} \longrightarrow \max
$$

Column j

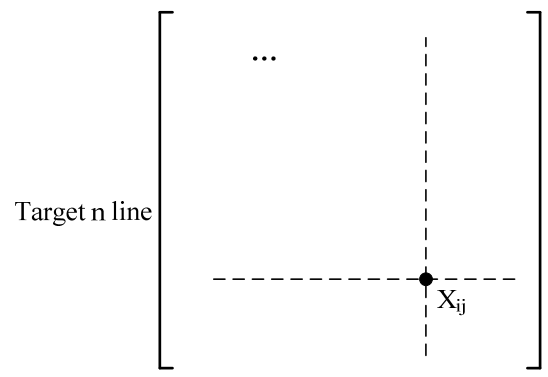

Line i

Fire unit $\mathrm{m}$ column

Coefficient matrix

Column $j$

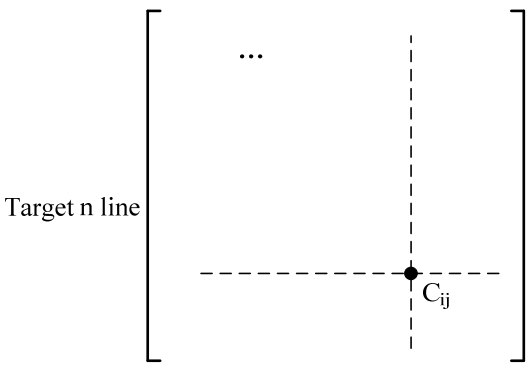

Line $\mathrm{i}$

Fire unit $\mathrm{m}$ column

Weight matrix

Column $\mathrm{j}$

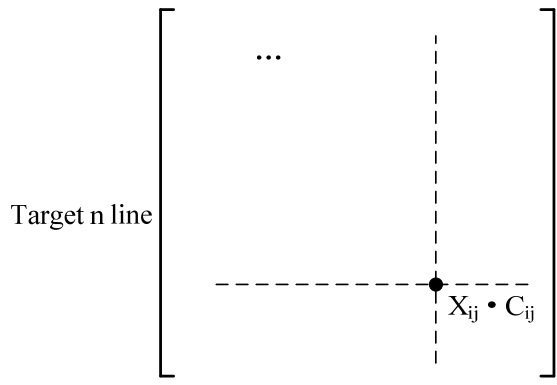

Line i

Fire unit $\mathrm{m}$ column

Weighting matrix 
Integer programming assignment rule:

Prerequisite: Fire units more than the target number

(1) The coefficient matrix has at least one $X i j=1$ per line, unless the line is $\max _{i}\left\{C_{i j}\right\}<\delta$.

(Meaning: Each target is attacked by at least one fire unit, unless the gain is too small.)

(2) The coefficient matrix has at least one $\mathrm{Xij}=1$ per column, unless the column is $\max _{j}\left\{C_{i j}\right\}<\delta$;

(Meaning: Each fire unit attacks at least one target, unless the gain is too small.)

(3) Under the conditions (1) (2), Xij in the coefficient matrix is equal to 1 does not exceed $\mathrm{P}(\mathrm{p}>1)$; p)

(Meaning: the total shooting relationship is not more than

(4) Under the above conditions, seeking the maximum value of the weighting matrix $\sum_{i=1}^{\mathrm{n}} \sum_{j=1}^{\mathrm{m}} X_{i j} C_{i j}$ total gain.

(Meaning: seeking the maximum benefit when meet the constraints)

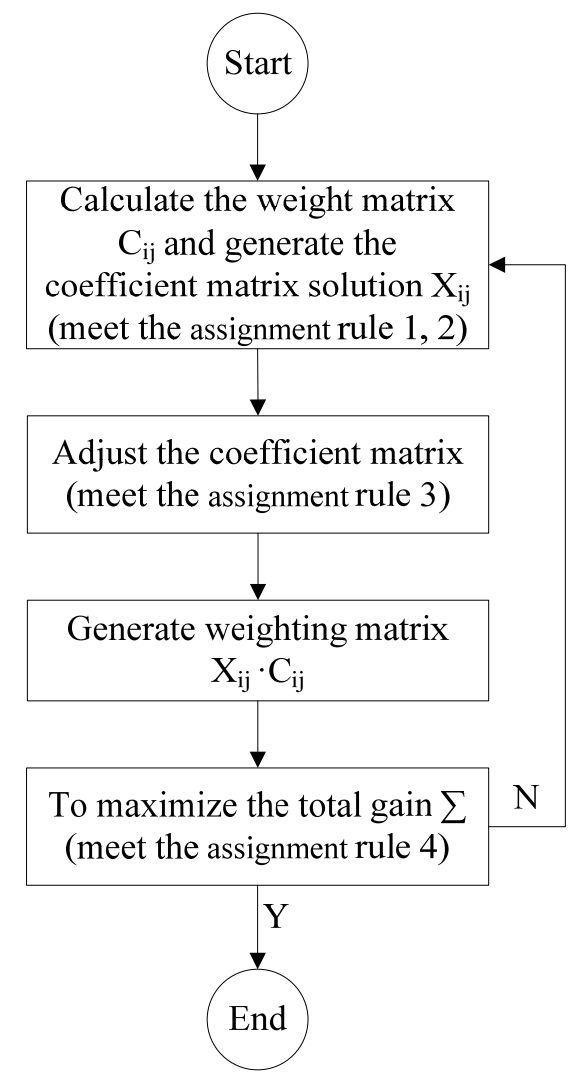

FIGURE II. INTEGER PROGRAMMING FLOW CHART.

\section{OTHER PROBLEM}

A. Each Fire Unit Determines the Order of Shooting (MultiTarget Case)

Elements in the Ej, according to the order $C_{i j}=A_{i j} \cdot B_{i j}$ from the big to small, that is, firing order.

\section{B. Weapons and Ammunition Selection}

According to the target type, the status of the unit and ammunition stock, determine the weapons and ammunition, the first selection is the best shooting effect (by the corresponding table).

\section{Shooting Opportunity}

Firing before enemy, which lead a larger destroyed probability. Understanding of the operational personnel is helpful also.

\section{REFERENCES}

[1] WANG Jian-fei, AN Fei, MING An-qing, "Research on the Target Assignment," FIRE CONTROL \& COMMAND CONTROL: 2008, 33(z1).

[2] Wang Zheng-yuan, Tan Yue-jin, "A Solution to Dynamic Weapontarget Assignment in the Tank Warfare," JOURNAL OF NATIONAL UNIVERSITY OF DEFENSE TECHNOLOGY: 2003, 25(6).

[3] CHEN Ying-wu, CAI Huai-ping, XING Li-ning, "An Improved Algorithm of Policies Optimization of Dynamic Weapon Target Assignment Problem," SYSTEMS ENGINEERING - THEORY \& PRACTICE: 2007, 27(7).

[4] WANG Wei, CHENG Shu-chang, ZHANG Yu-zhi, "Research on approach for a type of weapon target assignment problem solving by genetic algorithm," SYSTEMS ENGINEERING AND ELECTRONICS: 2008, 30(9).

[5] LI Yong-jun, HUANG Zhuo, GUO Bo, "Review of Weapon-Target Assignment Problem," ORDNANCE INDUSTRY AUTOMATION: 2009, 28(11).

[6] Han Zhi-jun, Xu Ke-hu, Li Meng, "Simulation Study on the CGF Entities of Tank Element," JOURNAL OF SYSTEM SIMULATION: 2004, 16(7). 